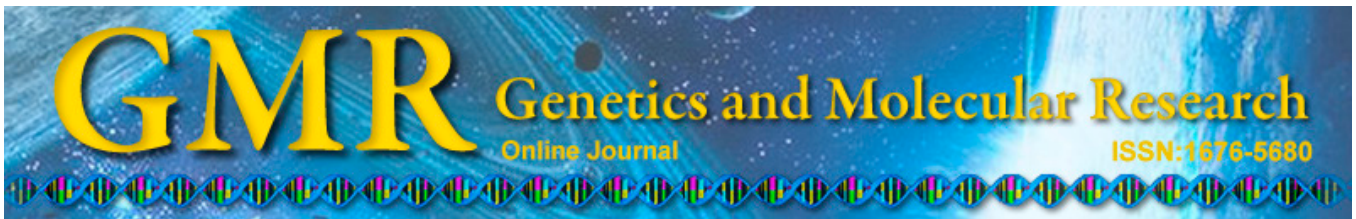

\title{
Molecular cloning, characterization and expression analysis of $W A G-1$ in the pistillody line of common wheat
}

\author{
Q.H. Wang, Z.J. Yang, S.H. Wei, Z.Y. Jiang, Y.F. Yang, Z.S. Hu, \\ Q.X. Sun and Z.S. Peng
}

The Ministry of Education Key Laboratory of Southwest China Wildlife Resources Conservation, College of Life Science,

China West Normal University, Nanchong City, Sichuan, China

Corresponding author: Z.S. Peng

E-mail:pzs8833@163.com

Genet. Mol. Res. 14 (4): 12455-12465 (2015)

Received February 4, 2015

Accepted June 8, 2015

Published October 16, 2015

DOI http://dx.doi.org/10.4238/2015.October.16.12

\begin{abstract}
Wheat $W A G-1$ is a C-class MADS-box gene, which is orthologous to AGAMOUS in Arabidopsis. In this study, we report the cloning, characterization, and expression patterns of $W A G-1$ in the pistillody mutant HTS- 1 and its sib-line CSTP. The cDNA of $W A G-1$ was found to be $765 \mathrm{bp}$ in length, which was equal to the length of its open reading frame, encoding 254 amino acids. The location of $W A G$ 1 revealed that it has three homologous genes from the short arm of chromosome 1A, 1B, and 1D. Their genomic sequences were determined to be 5864, 6454, and 6447 bp long, respectively, and possessed seven exons and six introns. Young spikes from HTS- 1 contained higher levels of $W A G-1$ transcript than did those from CSTP, and the transcript levels in the young spikes (7-10 mm in length) of HTS-1 increased 3.3fold relative to those of the CSTP line. The transcript level in the pistil and pistil-like stamens of HTS-1 was over 2-fold higher than that in the
\end{abstract}


stamens of CSTP, and expression in the pistil-like stamens of HTS-1 was slightly higher than that in its pistils. These data provide a basis for future research into the function of $W A G-1$, and offer further insight into the molecular mechanism of the pistillody mutation in common wheat.

Key words: $W A G-1$; Cloning; Pistillody line; Expression pattern; Wheat

\section{INTRODUCTION}

Floral organ formation has been the subject of intensive study for over 20 years, particularly in the model dicot species Arabidopsis thaliana and Antirrhinum majus. These studies have led to the establishment of a general model for the development of floral organs in higher plants. The so-called ABCDE model of flower development proposes that floral organ identity is defined by five classes of homeotic genes, named A, B, C, D, and E (Rijpkema et al., 2010). According to the floral quartet models of floral organ specification (Smaczniak et al., 2012), the A- and E-class protein complex develops sepals as the ground-state floral organs in the first floral whorl. The A-, B- and E-class protein complex specifies petals in the second whorl, the B-, C-, and E-class protein complex specifies stamens in the third whorl, and the $\mathrm{C}$ - and E-class protein complex specifies carpels in the fourth whorl. Cloning of ABCDE homeotic genes in Arabidopsis showed that they encode MADS-box transcription factors except for the class A gene, APETALA2 (AP2) (Jofuku et al., 1994). On the basis of the amino acid sequence of MADS-box proteins, two typical structure characteristics, namely SRF (type I) and MEF2 (type II), were included. MADS-box genes in higher plants are mostly type II proteins (Münster et al., 1997). All of type II proteins are composed of MADS-box domain, I domain, $\mathrm{K}$ domain and $\mathrm{C}$ domain structure, which are also called MIKC-type genes (Münster et al., 1997; Nam et al., 2003). These genes not only play a significant role in individual plant development, but they also have vital effects on floral organ formation (Alvarez-Buylla et al., 2000; Ng and Yanofsky, 2001).

The wheat $A G$ ortholog wheat $A G A M O U S 1$ (WAG-1) is a C-class gene (Meguro et al., 2003; Hirabayashi and Murai, 2009). MADS-box genes of the AG group play a central role in the identification, formation, and development of gynoecium. Additionally, the AG family are also involved in the negative regulation of class A MADS-box genes (Paolacci et al., 2007). Southern blot analysis revealed that wheat $W A G-1$ contains three homologous genes, which are located on homologous chromosomes 1A, 1B, and 1D, respectively. Two different transcripts $(1.1$ and $1.3 \mathrm{~kb})$ were found at the booting and heading stages. It is worth noting that the 1.1-kb WAG-1 transcript accumulates in both the reproductive (pistil and stamen) nonreproductive (palea and lemma) parts of the plant, whereas the 1.3-kb WAG-1 transcript exists only in pistil-like stamens of homoplasmic and alloplasmic wheat. These findings indicate that the product of the 1.3-kb WAG-1 transcript is involved in pistil development, and it is associated with pistillody caused by nuclear-cytoplasm interactions in alloplasmic wheat (Meguro et al., 2003).

In the present study, we re-cloned, characterized, and phylogenetically analyzed $W A G-1$ in the pistillody mutant HTS- 1 and its sib-line Chinese Spring TP (CSTP). We also examined the expression patterns of this gene in different developmental stages of young spikes, including the pistils (P), pistillody stamens (PS), and stamens (S). 


\section{MATERIAL AND METHODS}

\section{Plant materials}

HTS-1 is a new pistillody common wheat mutant found in CSTP. CSTP is a nearisogenic line of the common wheat variety CS that carries the Pis 1 gene derived from the TP mutant (Yang et al., 2012), and was used as a non-pistillody control in this study. HTS-1 was selected during the development process of CSTP. These are sib-lines that show a similar phenotype except for pistillody. They were grown in an experimental field at the China West Normal University, Nanchong, China. Young spikes at the reproductive stage were collected and examined with a stereomicroscope (Olympus SZX9, Japan) to accurately determine the development stage. Flag leaves were selected for DNA analysis and subsidiary pistil spikes in the primordial stage of differentiation (spikes $\sim 7-10 \mathrm{~mm}$ ) were used as a source of RNA for cloning the cDNA and sequencing WAG-1. Young spikes of HTS-1 and CSTP at various development stages (spikes 2-5, 5-7, 7-10 mm in length), P and PS in HTS-1, and S in CSTP at the heading stage were selected for real-time PCR analysis. All young spikes were protected in RNA preservative fluid, snap frozen in liquid nitrogen $\left(-196^{\circ} \mathrm{C}\right)$, and subsequently placed in an ultra-low temperature freezer $\left(-70^{\circ} \mathrm{C}\right)$.

\section{DNA and RNA extraction}

Genomic DNA was isolated from fresh flag leaves of CSTP, according to methods described by Porebski et al. (1997). DNA was then dissolved in Tris-EDTA buffer and maintained at $-20^{\circ} \mathrm{C}$. Total RNA was extracted from young spikes as described by Manickavelu et al. (2007). The RNA was dissolved in RNase-free double-distilled water and stored at $-70^{\circ} \mathrm{C}$. The quality of RNA was confirmed by $1.0 \%(\mathrm{w} / \mathrm{v})$ agarose gel electrophoresis, the concentration of nucleic acid was determined spectrophotometrically with a Photometer (Nanodrop2000C, Thermo, USA) based on the 260/280-nm absorbance ratio, and samples were diluted to a concentration of $50 \mathrm{ng} / \mu \mathrm{L}$ for PCR amplification.

\section{Primer design, RT-PCR, cloning, and sequencing}

PCR primers were designed using Primer Premier 5.0 based on the mRNA sequence of WAG-1 from Triticum aestivum (GenBank accession No. AB084577.1). The specific primers used to amplify genomic and cDNA sequences are shown in Table 1.

Table 1: Primer sequences used in this study.

\begin{tabular}{llll}
\hline PCR target & Primer & Forward & Reverse \\
\hline Genomic and cDNA & WAG-1-C & 5'-ATGGTGAAGGAGTCTGCGTC-3' & 5'-TCACCTTCCAACTGAGTTCAAAC-3' \\
Real-time PCR & WAG-1-Q & 5'-AACAGGACTCTAATAGGCGATACAAT-3' & 5'-ATTCGTTCTTTCTTGCTCTAATCTTT-3' \\
Internal control & Ubiquitin & 5'-AAGGCGAAGATCCAGGACAAG - 3' & 5'-TGGATGTTGTAGTCCGCCAAG - 3' \\
& Actin & 5'-ACGCTTCCTCATGCTATCCTTC - 3' & 5'-ATGTCTCTGACAATTTCCCGCT - 3' \\
\hline
\end{tabular}

Total RNA was synthesized into first-strand cDNA using the Super Smart cDNA Synthesis Kit according to the manufacturer instructions (Clontech, Palo Alto, CA, USA). Standard PCR amplification was also performed using cDNA from the young spikes as a template. 
The PCR products were separated by electrophoresis on $1.0 \%$ agarose gels with a molecular size marker (DL2000, TaKaRa, Dalian, China), followed by sequencing of all amplified bands by Taihe Biotechnology Co., Ltd. (Beijing, China).

The genomic sequence of $W A G-1$ was amplified using touchdown-PCR under the following conditions: predenaturation at $94^{\circ} \mathrm{C}$ for $5 \mathrm{~min}$, followed by 35 cycles of denaturation at $94^{\circ} \mathrm{C}$ for $30 \mathrm{~s}$, annealing at $58^{\circ} \mathrm{C}$ for $30 \mathrm{~s}$, extension at $72^{\circ} \mathrm{C}$ for $6 \mathrm{~min}$, and final extension at $72^{\circ} \mathrm{C}$ for $10 \mathrm{~min}$. The amplified fragment was also purified and sequenced by Taihe Biotechnology Co., Ltd.

\section{Molecular characterization of $W A G-1$}

Sequence data were analyzed with the GenScan software (http://genes.Mit.Edu/ GENSCAN.html). The open reading frame (ORF) of the cDNA sequence was identified using ORF finder software (http://www.ncbi.nlm.nih.gov/gorf/gorf.html). The cDNA sequences of $W A G-1$ with the draft sequence of the bread wheat genome were compared using the BLAST software (https://urgi.versailles.inra.fr/blast/blast.php). For homology analysis, the amino acid sequence of $W A G-1$ was compared with the sequences of other species using BLAST 2.1 (http://www.ncbi.nlm.nih.gov/blast/). Multiple sequence alignment was performed using the DNAstar Lasergene and DNAMAN 6.0 software. The structure of the $W A G-1$ genomic sequences was analyzed via the GSDS software (http://gsds1.cbi.pku.edu.cn).

\section{Multiple sequence alignment and phylogenetic analysis}

The deduced amino acid sequence of $W A G-1$ was aligned with that of C-class MADSbox genes reported for other species using the ClustalW program (Thompson et al., 1994). Neighbor-joining trees of the C-class MADS-box genes were generated with the MEGA software version 5.0 (Saitou and Nei, 1987; Tamura et al., 2007) with1000 bootstrap replicates. Gaps were treated as missing values. Amino acid sequences were obtained from the NCBI, and each GenBank accession No. is as follows: T aestivum L. WAG-2: BAH56656.1, AGL39: ABF57939.1, TaAG-1: CAM59041.1, WM29A: CAM59076.1, WM29B: CAM59077.1, Hordeum vulgare HvAG1: AAL93196.1, HvAG2: AAL93197.1, Oryza sativa L. OsMADS3: ACY26070.1, OsMADS58: BAE54300.1, Zea mays ZAG1: AAA02933.1, ZMM2: CAA57074.1, A. thaliana (L.) Heynh AG: NP 567569.3, A. majus PLE: AAB25101.1, AmFAR: CAB42988.1, Petunia hybrida FBP6: CAA48635.1, and Carica papaya CpPLE: ABR68012.1.

\section{Real-time PCR}

Total RNA was isolated from spikes of the sister wheat lines CSTP and HTS-1 at various development stages (2-5, 5-7, 7-10 $\mathrm{mm}$ in length). cDNA was synthesized using a PrimeScript Perfect real-time RT reagent kit (TaKaRa, Dalian, China), following the manufacturer protocol. The primers for WAG-1-Q (Table 1) were designed using Prime Primer Premier 5.0 software to amplify short fragments of $W A G-1$. Real-time assays were performed with SYBR green (TaKaRa) using a Bio-Rad CFX96 real-time PCR platform. All samples were analyzed in triplicate and the fold change in the number of RNA transcripts was calculated 
using the $2^{-\Delta \Delta C t}$ method (Livak and Schmittgen, 2001) with the wheat housekeeping genes ubiquitin (DQ086482) and actin (AB181911) acting as internal controls (Hama et al., 2004; Yamada et al., 2009).

\section{RESULTS}

\section{cDNA cloning and chromosomal location}

cDNA that was amplified from young spikes of CSTP and HTS-1 plants using the DL primer pair yielded a fragment of $\sim 760 \mathrm{bp}$. The PCR product was cloned into the PMD-19T vector and 15 clones were sequenced. Three distinct sequences of 765 bp were identified, indicating that the band of $\sim 760 \mathrm{bp}$ included comigrating cDNA of three homologous genes, which shared $99.36 \%$ homology. BLAST searches identified the predicted 765-bp sequence (GenBank accession No. AB084577.1), which had up to 100\% homology with the open reading frame (ORF) of $W A G-1$, indicating that the 765-bp band belongs to $W A G-1$. The cloned sequences were found to be homologous via the BLAST software (https://urgi.versailles.Inra. fr/blast/blast.php), and were located on the short arm of chromosome 1A, 1B, and 1D, respectively. The ORF of $W A G-1$ was 765-bp long and encoded a deduced protein of 254 amino acids. Three different amino acid sites exist (15th, 236th, and 240th) on the short arm of WAG1-1A, WAG-1-1B, WAG-1-1D chromosome, respectively (Figure 1).

BLAST searches of the $W A G-1$ genomic sequence against the draft sequence of the bread wheat genome database (https://urgi.versailles.inra.fr/blast/blast.php) (IWGSC, 2014) revealed that WAG-1-1AS, WAG-1-1BS, and WAG-1-1DS showed 100\% homology to the IWGSC_chr1AS_ab_k71_contigs_longerthan_200_3283142, IWGSC_chr1BS_ab_k71_contigs longerthan_200_3430623, and IWGSC_chr1DS ab_k71_contigs_longerthan_200_1915282, respectively. It can therefore be deduced that the three homologous genes $W A \bar{G}-1-\overline{1} A S, W A G$ $1-1 B S$, and $W A G-1-1 D S$ are located on the short arm of chromosome $1 \mathrm{~A}, 1 \mathrm{~B}$, and $1 \mathrm{D}$, respectively.

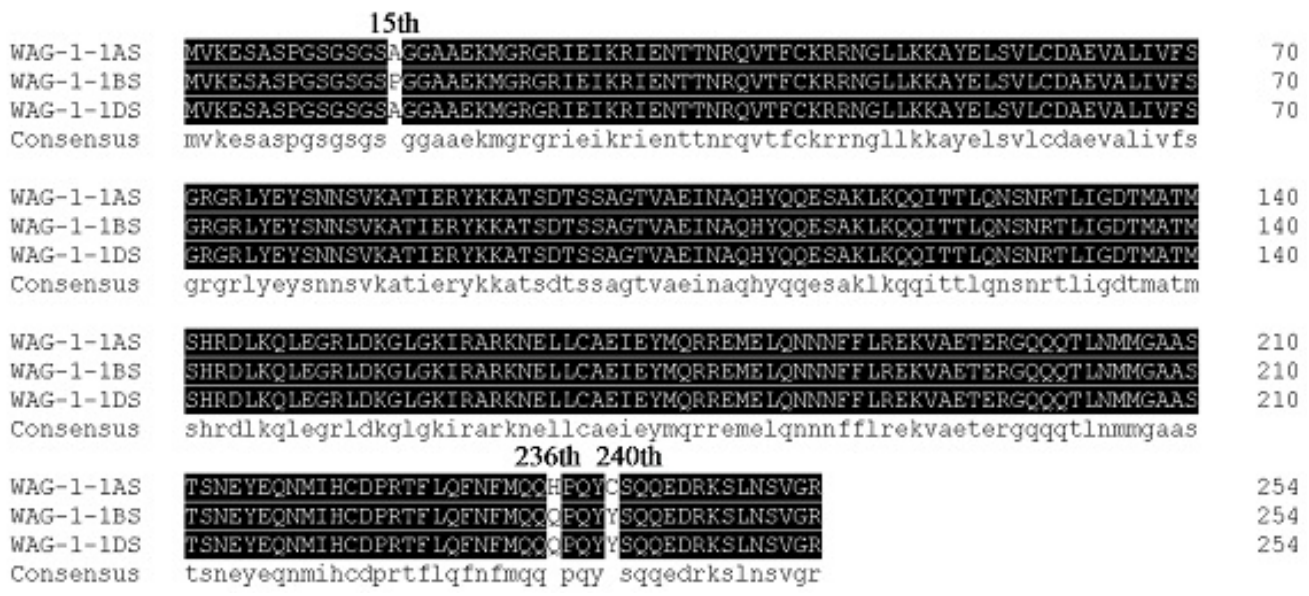

Figure 1. Alignment of the three homologous sequences of WAG-1 (WAG-1-1AS, WAG-1-1BS, WAG-1-1DS), located on the short arm of chromosome 1A, 1B, and 1D. 


\section{Structure of the $W A G-1$ genomic sequences}

WAG-1-1A, WAG-1-1B, and WAG-1-1D genomic sequences were 5864, 6454, and 6447 bp long, respectively. Alignment and comparison with the corresponding cDNA sequences revealed the complex structure of the three homologous genes, which consisted of seven exons and six introns (Figure 2).

The length and sequence of the six introns varied markedly. The length of intron VI different markedly, and was 3003, 3734, and 3848 bp in WAG-1-1A, WAG-1-1B, and WAG-1$1 D$, respectively, and was longer than the other five introns. Intron $\mathrm{V}$ was the second longest and consisted of 1580,1542 , and $1421 \mathrm{bp}$. Intron IV was 100-bp long in all three genes. Intron III was 88,92 , and 89 bp long in $W A G-1-1 A, W A G-1-1 B$, and $W A G-1-1 D$, respectively, and was the shortest of the six introns. Intron I was 226, 123, and 127 bp long in WAG-1-1A, WAG$1-1 B$, and $W A G-1-1 D$, respectively, and Intron II was 102, 98, and 97 bp long, respectively.

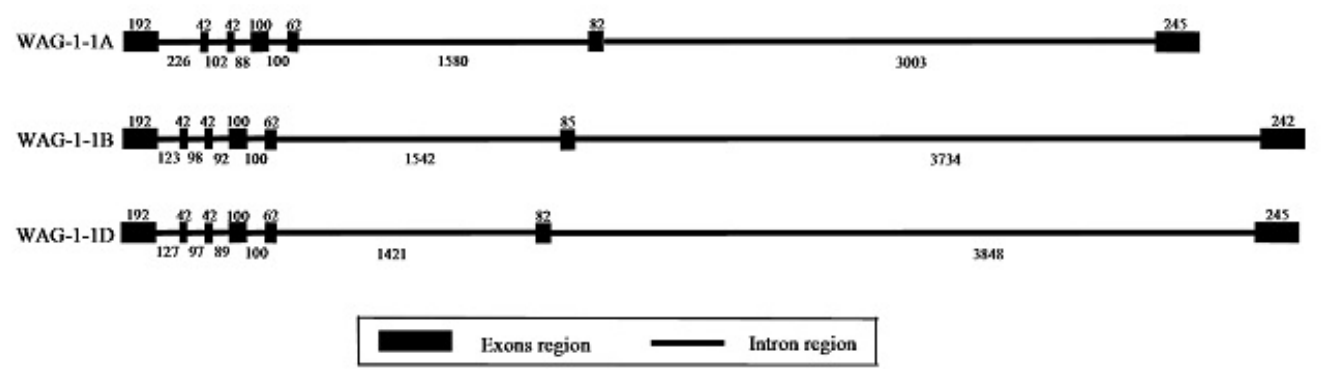

Figure 2. Schematic representation of the $W A G-1$ gene.

\section{Sequences alignment of $W A G-1$ and other C-class genes}

Multiple sequence alignment revealed that $W A G-1$ shows relatively high homology with $H v A G 2$ and OsMADS58 (86.61 and 73.82\%), whereas the lowest homology was observed with $Z M M 2(53.33 \%)$. Additionally, $W A G-1$ was found to have similarly homology with $H v A G 1, O s M A D S 3$, and $Z A G 1(65.89,65.64$, and $64.01 \%$, respectively) from gramineous barley, rice, and maize. As shown in Figure 3, WAG-1 is a MIKC-type gene, which contains a MADS-MEF2 domain, a K-box domain, an $A G$ motif I, and an $A G$ motif II.

\section{Phylogenetic analysis of $W A G-1$ and other C-class genes}

C-class MADS-box genes from T. aestivum L. (WAG-2, AGL39, TaAG-1, WM29A, and $W M 29 B), H$. vulgare (HvAG1 and HvAG2), O. sativa L. (OsMADS3 and OsMADS58), Z. mays (ZAG1 and ZMM2), A. thaliana (AG), A. majus (PLE and AmFAR), P. hybrida (FBPO), and $C$. papaya $(C p P L E)$ were used to construct a phylogenetic tree. The phylogenetic tree obtained with the neighbor-joining program using the MEGA software and 1000 bootstrap replicates is shown in Figure 4. In the present study, phylogenetic analysis shows that three types of C-class MADS-box genes exist in plants, namely AG-like, euAG, and PLE. The monocots, such as wheat, rice, maize, and barley were grouped into AG-like, whereas the other dicots 
were grouped into euAG or PLE. These C-class MADS-box genes formed two well-resolved clades (WAG-1 clades and $W A G-2$ clades) in gramineous plants. WAG-1 (WAG-1-1AS, WAG1-1BS, and $W A G-1-1 D S$ ), TaAG-1, HvAG2, OsMADS58, and ZAG1 were grouped into WAG-1 clades, whereas $W A G-2, W M 29 A, W M 29 B, A G L 39, H v A G 1$, and $O S M A D S 3$ were classified into $W A G-2$ clades.

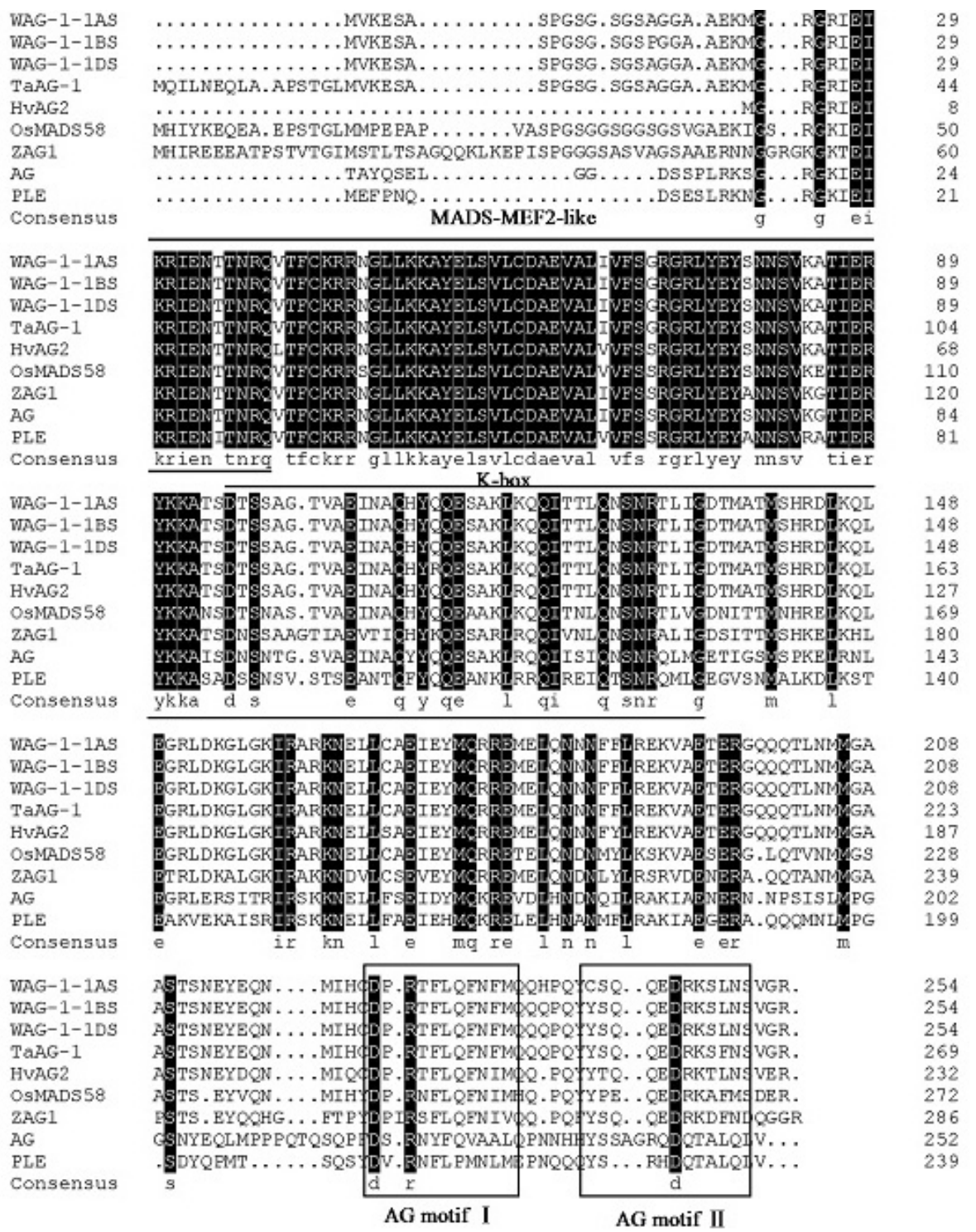

Figure 3. Amino acid sequence alignment of WAG-1(WAG-1-1AS, WAG-1-1BS, WAG-1-1DS) with TaAG-1 (Triticum aestivum), HvAG2 (Hordeum vulgare), OsMADS58 (Oryza sativa), ZAG1 (Zea mays), AG (Arabidopsis thaliana), and PLE (Antirrhinum majus). The MADS-MEF2 domain, K-box domain, $A G$ motif I and $A G$ motif II marked with a thick, thin line, and a square frame, respectively. 


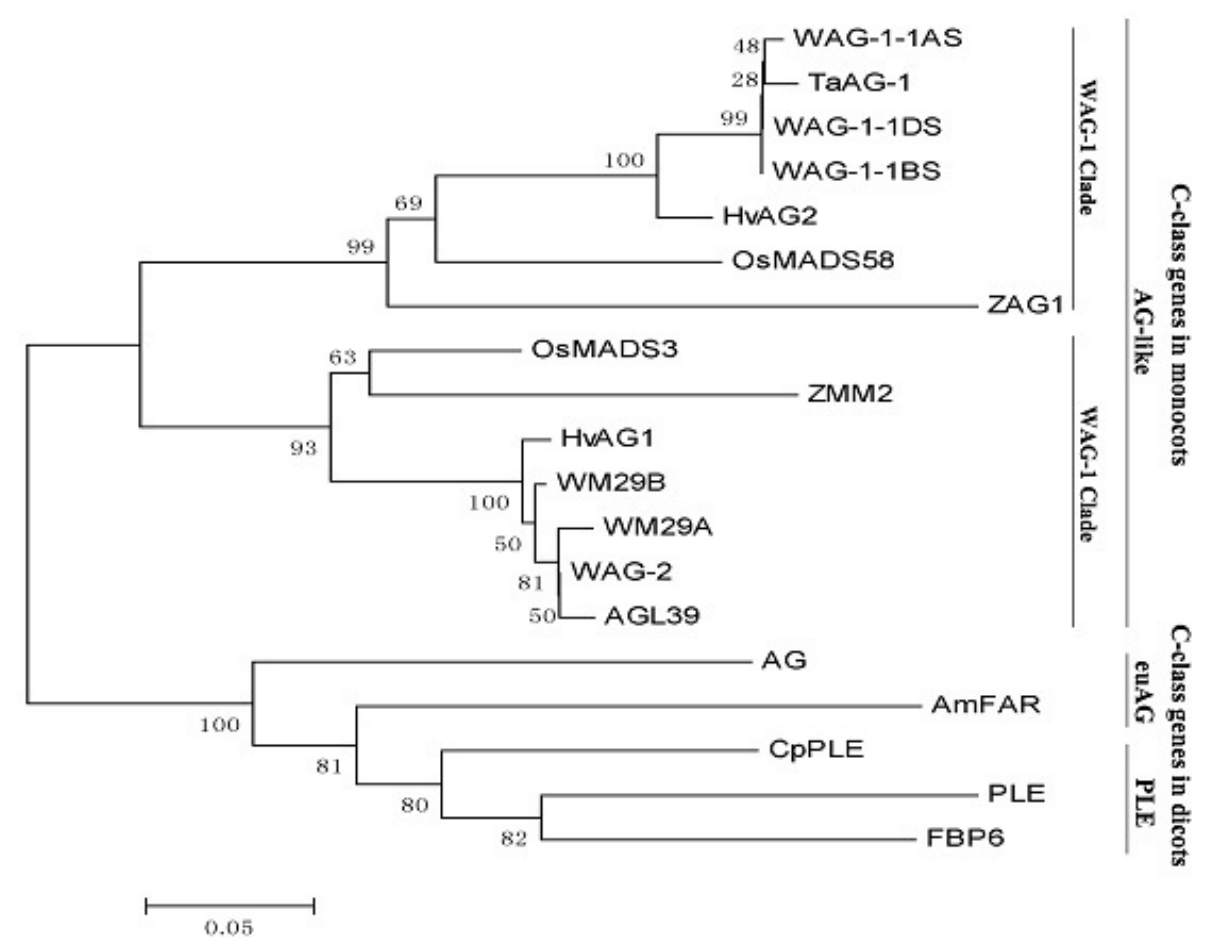

Figure 4. Phylogenetic tree of WAG-1 (WAG-1-1AS, WAG-1-1BS, WAG-1-1DS) and other C-class homologous genes in different plant species. The numbers at the nodes indicate bootstrap values.

\section{$W A G-1$ expression in developing spikes and different tissues}

Real-time PCR was used to study the pattern of $W A G-1$ expression in different wheat materials during the developmental stages of young spikes. As shown in Figure 5a, WAG-1 expression was low in young spikes of CSTP at stages 2-5, 5-7, and 7-10 $\mathrm{mm}$, and the levels were similar at all stages. However, the transcript levels in young spikes of HTS-1 at stages 2-5, 5-7, and 7-10 mm were 1.4-, 2.6-, and 3.3-fold higher than in CSTP at the same stage. Therefore, the difference in expression between CSTP and HTS-1 increases gradually during spike development.

Similarly, real-time PCR was also used to analyze the pattern of WAG-1 expression in different tissues. Figure $5 \mathrm{~b}$ shows that the transcript levels in pistils and pistil-like stamens in HTS-1 were more than 2-fold higher than that in the stamens of CSTP, and the pistil-like stamens of HTS-1 contained slightly higher levels than its pistils.

\section{DISCUSSION}

Previously, WAG-1 had been cloned in Three Pistil (TP) and CS, based on the cDNA sequence obtained from the NCBI, and was designed as $W A G-1$ (Wu, et al., 2012). In the present study, the cDNA and genome sequence of $W A G-1$ were cloned successfully from wheat CSTP and its pistillody mutant (HTS-1), and the results showed that there is no variation in 
the cDNA sequences from CSTP and HTS-1, that all sequences are 765-bp long, and that three homologous $W A G-1$ genes exist, which are $W A G-1-1 A S, W A G-1-1 B S$, and $W A G-1-1 D S$, and are located on the short arm of chromosome 1A, 1B, and 1D, respectively. The nucleotide coding sequences of the three homologous genes were highly conserved, with homologies of close to $100 \%$ noted. Additionally, only three subtle differences were observed in the three deduced amino acid sequences (Figure 1), which occur at the 15th, 236th, and 240th amino acid sites. The putative $W A G-1$ protein contained 254 amino acids, which consisted of the highly conserved MADS-MEF2 type protein domain, and the relatively highly conserved Kbox protein domain, and the non-conserved protein domain in the C-terminal region. Despite variation in the nucleotide and amino acid sequences of C-class genes from different plants, these proteins typically contained two protein motifs, $A G$ motif I and $A G$ motif II (Figure 3), and are both $A G$-type genes. The genomic sequences of $W A G-1-1 A, W A G-1-1 B$, and $W A G-1$ $1 D$ contained seven exons and six introns, all of which (except for intron IV) varied in length and sequence composition.

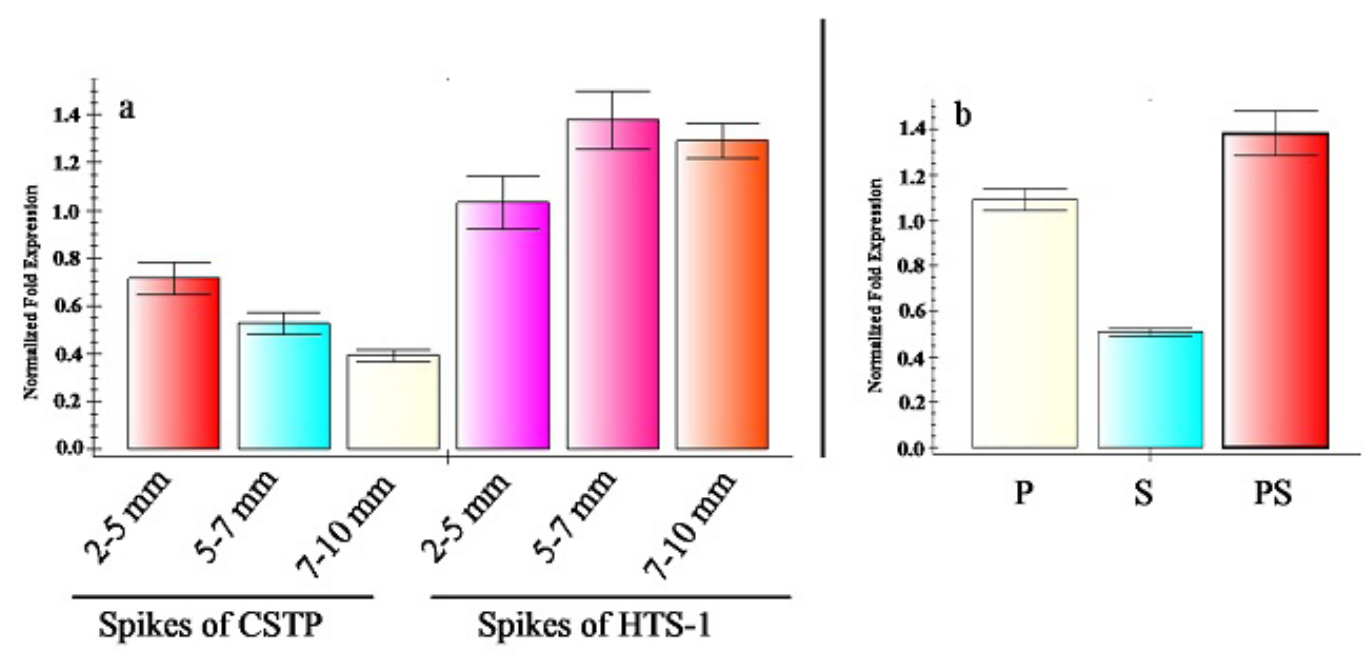

Figure 5. WAG-1 expression assessed by real-time PCR. Total RNA was isolated from young spikes of CSTP and HTS- 1 at various development stages and from different tissues. Ubiquitin and actin genes were used as internal controls. The transcript levels are shown as relative values and the columns represent the means + SEM of three replicates. $\mathrm{P}=$ pistils, $\mathrm{S}=$ stamens, $\mathrm{PS}=$ pistillody stamens.

C-class MADS-box genes can be classified into three types, namely euAG, PLE, and $A G$-like. Most dicots could be grouped with the former two, and most monocots were classified as the latter. $A G$-like group $C$-class genes from monocots contain two clades, $W A G-1$ and $W A G$-2 clade, WAG-1 (WAG-1-1AS, WAG-1-1BS, WAG-1-1DS) formed the WAG-1 clade together with wheat $T a A G-1$, barely $H v A G 2$, maize $Z A G 1$, and rice $O S M A D S 58$, whereas $W A G-2$ grouped into the $W A G-2$ clade with wheat $A G L 39, W M 29 A$, and $W M 29 B$, and barley $H v A G 1$, maize $Z M M 2$ and rice $O S M A D S 3$ (Figure 4). Using the deduced amino acid sequences, phylogenetic analyses showed that $W A G-1$ and $W A G-2$ are orthologs of the rice $A G$-type genes, $O S$ MADS58 and OSMADS3, respectively (Hirabayashi and Murai, 2009; Wei et al., 2011). WAG-1 
and $W A G-2$ have also been referred to as TaAG-2 and TaAGL39, respectively (Zhao et al., 2006; Paolacci et al., 2007). Phylogenetic analysis revealed that $W A G-1$ clustered more closely with $H$. vulgare $H v A G 2$. Furthermore, alignment analysis demonstrated that $W A G-1$ shared higher identity with $H v A G 2(86.61 \%)$ than with other C-class $A G$-type genes. These findings suggest that $W A G-1$ and $H v A G 2$ are orthologs of O. sativa OsMADS58 and may have similar roles. The $O$. sativa OsMADS58 gene is homeotic with $O$. sativa OSMADS3, and the two C-class genes may have arisen by gene duplication before the divergence of rice and maize (Z. mays), and were partially subfunctionalized during rice evolution (Yamaguchi et al., 2006). Similarly, it is suggested that $W A G-2, W M 29 A$, and $W M 29 B$ could be three homologous genes located on three homologous chromosomes, and are likely to be orthologs of OsMADS3 with HvAG1.

Expression analysis showed that the levels of WAG-1 mRNA were higher at every stage of HTS-1 young spikes than in CSTP (Figure 5a). Similarly, the level of expression in PS was higher than that in P, and was lowest in S (Figure 5b). In the HTS-1 pistillody mutant, homeotic transformation of stamens into pistil-like structures, means that it has at least one more pistil per floret than CSTP (Yang et al., 2012). Based on these observations, we suggest that the pattern of WAG-1 expression may contribute to pistil development and could be functionally important in defining the morphology of the pistillody mutant. According to the ABCDE model, the B-, C-, and E-class genes determined the presence of stamens in the third whorl, and the deficiency of B-class MADS-box genes resulted in homeotic change of S into carpels in Arabidopsis (Jack et al., 1992; Goto and Meyerowitz, 1994) and Antirrhinum (Sommer et al., 1990; Tröbner et al., 1992). Additionally, the C-class genes could negatively regulate the A-class genes (Coen and Meyeroitz, 1991). These findings strongly indicate that the ABCDE model of floral organ development in dicots can be extended to rice and maize, and probably to wheat. However, what is the functional relationship between the B- and Cclass genes? Whether the A-class genes affect the C-class genes on expression patterns? The detailed function of $W A G-1$ require further study.

\section{Conflicts of interest}

The authors declare no conflict of interest.

\section{ACKNOWLEDGMENTS}

Research supported by the National Natural Science Fund of China (grant \#31301319 and \#31540041) and the Key Project Fund of Educational Ministry (grant \#211164), which was also supported by the Students' Science and Technology Innovation Fund of China West Normal University (grant \#201410638016).

\section{REFERENCES}

Alvarez-Buylla ER, Liljergren SJ, Pelaz S, Gold SE, et al. (2000). MADS-box gene evolution beyond flowers: expression in pollen, endosperm, guard cells, roots and trichomes. Plant J. 24: 457-466.

Coen ES and Meyeroitz EM (1991). The war of the whorls: genetic interactions controlling flower deelopment. Nature 353: 31-37.

Goto K and Meyerowitz EM (1994). Function and regulation of the Arabidopsis oral homeotic gene PISTILLATA. Genes Dev. 8: 1548-1560. 
Hama E, Takumi S, Ogihara Y and Murai K (2004). Pistillody is caused by alterations to the class-B MADS-box gene expression pattern in alloplasmic wheats. Planta 218: 712-720.

Hirabayashi C and Murai K (2009). Class C MADS-box gene AGAMOUS was duplicated in the wheat genome. Wheat Inf. Serv. 107: 13-16.

Jack T, Brockman LL and Meyerowitz EM (1992). The homeotic gene APETALA3 of Arabidopsis thaliana encodes a MADS box and is expressed in petals and stamens. Cell 68: 683-697.

Jofuku KD, den Boer BG, Van Montagu M and Okamuro JK (1994). Control of Arabidopsis flower and seed development by the homeotic gene APETALA2. Plant Cell 6: 1211-1225.

Livak KJ and Schmittgen TD (2001). Analysis of relative gene expression data using real time quantitative PCR and the $2^{-\Delta \Delta C t}$ method. Methods 25: 402-408.

Manickavelu A, Kambara K, Mishina K and Koba T (2007). An efficient method for purifying high quality RNA from wheat pistils. Colloids Surf. B: Biointerfaces 54: 254-258.

Meguro A, Takumi S, Ogihara Y and Murai K (2003). WAG, a wheat AGAMOUS homolog, is associated with development of pistil-like stamens in alloplasmic wheats. Sex. Plant Reprod. 15: 221-230.

Münster T, Pahnke J, Di Rosa A, Kim JT, et al. (1997). Floral homeotic genes were recruited from homologous MADSbox genes preexisting in the common ancestor of fems and seed plants. Proc. Natl. Acad. Sci. U. S. A. 94: 2415-2420.

Nam J, Kim J, Lee S, An J, et al. (2003). Type I MADS-box genes have experienced faster birth-and-death evolution than type II MADS-box genes in angiosperms. Proc. Natl. Acad. Sci. U. S. A. 101: 1910-1915.

$\mathrm{Ng} \mathrm{M}$ and Yanofsky MF (2001). Function and evolution of the plant MADS-box gene family. Nat. Rev. Genet. 2: 186-195.

Paolacci AR, Tanzarella OA, Porceddu E, Varotto S, et al. (2007). Molecular and phylogenetic analysis of MADS-box genes of MIKC type and chromosome location of SEP-like genes in wheat (Triticum aestivum L.). Mol. Genet. Genomics 278: 689-708.

Porebski S, Bailey LG and Baum BR (1997). Modification of a CTAB DNA extraction protocol for plants containing high polysacchharide and polyphenol components. Plant Mol. Biol. Rep. 15: 8-15

Rijpkema AS, Vandenbussche M, Koes R, Heijmans K, et al. (2010). Variations on a theme: Changes in the floral ABCs in angiosperms. Semin. Cell Dev. Biol. 21: 100-107.

Saitou N and Nei M (1987). The neighbor-joining method: A new method for reconstructing phylogenetic trees. Mol. Biol. Evol. 4: 406-425.

Smaczniak C, Immink RG, Angenent GC and Kaufmann K (2012). Developmental and evolutionary diversity of plant MADS-domain factors: Insights from recent studies. Development 139: 3081-3098.

Sommer H, Beltrân JP, Huijser P, Pape H, et al. (1990). Deficiens, a homeotic gene involved in the control of flower morphogenesis in Antirrhinum majus: the protein shows homology to transcription factors. EMBO J. 9: 605-613.

Tamura K, Dudley J, Nei M and Kumar S (2007). MEGA4: Molecular Evolutionary Genetics Analysis (MEGA) software version 4.0. Mol. Biol. Evol. 24: 1596-1599.

Thompson JD, Higgins DG and Gibson TJ (1994). CLUSTAL W: Improving the sensitivity of progressive multiple sequence alignment through sequence weighting, position-specific gap penalties and weight matrix choice. Nucleic Acids Res. 22: 4673-4680.

Tröbner W, Ramirez L, Motte P, Hue I, et al. (1992). GLOBOSA: a homeotic gene which interacts with DEFICIENS in the control of Antirrhinum oral organogenesis. EMBO J. 11: 4693-4704.

Wei S, Peng Z, Zhou Y, Yang Z, et al. (2011) Nucleotide diversity and molecular evolution of the $W A G-2$ gene in common wheat (Triticum aestivum L.) and its relatives. Genet. Mol. Biol. 34: 606-615.

Wu K, Peng ZS, Yang ZJ, Wei SH, et al. (2012). Differential expression of WAG1 from the common wheat Line Three Pistils and China Spring. J. China West Normal Univ. 33: 1-7.

Yamada K, Saraike T, Shitsukawa N, Hirabayashi C, et al. (2009). Class D and B (sister) MADS-box genes are associated with ectopic ovule formation in the pistil-like stamens of alloplasmic wheat (Triticum aestivum L.). Plant Mol. Biol. 71: $1-14$.

Yamaguchi T, Lee DY, MiyaoM, Hirochika H, et al. (2006). Functional diversification of the two C-class MADS box genes OSMADS3 and OSMADS58 in Oryza sativa. Plant Cell. 18: 15-28.

Yang ZJ, Peng ZS, Zhou YH, Peng LJ, et al. (2012). Evaluation on the genetic background of near isogenic lines for three pistils character by SRAP markers. J. Nucl. Agric. Sci. 26: 22-27.

Zhao T, Ni Z, Dai Y, Yao Y, et al. (2006). Characterization and expression of 42 MADS-box genes in wheat (Triticum aestivum L.). Mol. Genet. Genomics 276: 334-350. 